\title{
Applying DNA barcodes for identification of economically important species in Brassicaceae
}

\author{
X.Q. Sun ${ }^{1 *}$, Y.Q. Qu ${ }^{1 *}$, H. Yao², Y.M. Zhang ${ }^{1}$, Q.Q. Yan ${ }^{1}$ and Y.Y. Hang ${ }^{1}$ \\ ${ }^{1}$ Institute of Botany, Jiangsu Province and Chinese Academy of Sciences, Nanjing, \\ China \\ ${ }^{2}$ Institute of Medicinal Plant Development, Chinese Academy of Medical Sciences, \\ Peking Union Medical College, Beijing, China \\ *These authors contributed equally to this study. \\ Corresponding author: Y.Y. Hang \\ E-mail: hangyueyu@cnbg.net
}

Genet. Mol. Res. 14 (4): 15050-15061 (2015)

Received June 11, 2015

Accepted August 11, 2015

Published November 24, 2015

DOI http://dx.doi.org/10.4238/2015.November.24.13

ABSTRACT. Brassicaceae is a large plant family of special interest; it includes many economically important crops, herbs, and ornamentals, as well as model organisms. The taxonomy of the Brassicaceae has long been controversial because of the poorly delimited generic boundaries and artificially circumscribed tribes. Despite great effort to delimitate species and reconstruct the phylogeny of Brassicaceae, little research has been carried out to investigate the applicability and effectiveness of different DNA regions as barcodes - a recent aid for taxonomic identification - to identify economically important species in Brassicaceae. In this study, we evaluated the feasibility of five intensively recommended regions [ $r b c L$, matK, trnH-psbA, internal transcribed spacer (ITS), ITS2] as candidate DNA barcodes to discriminate economic species of Brassicaceae in China and try to establish a new digital identification method for economic plants of Brassicaceae. All sequences of 58 samples from 27 economic species (Brassicaceae) in China were assessed in the success rates of 
PCR amplifications, intra- and inter-specific divergence, DNA barcoding gaps, and efficiency of identification. Compared with other markers, ITS showed superiority in species discrimination with an accurate identification of $67.2 \%$ at the species level. Consequently, as one of the most popular phylogenetic markers, our study indicated that ITS was a powerful but not perfect barcode for Brassicaceae identification. We further discuss the discrimination power of different loci due to inheritance pattern, polyploidization and hybridization in species-specific evolution. Further screening of other nuclear genes related to species isolation as plant barcode candidates is also proposed.

Key words: matK; rbcL; trnH-psbA; ITS; ITS2; Brassicaceae

\section{INTRODUCTION}

Brassicaceae (Cruciferae or mustard family) is a large plant family with approximately 338 genera and 3,709 species widely distributed globally. It is of special interest, as it includes many economically important crops, herbs, ornamentals, and model organisms. The most important edible oil crop is canola or rapeseed (Brassica napus L.), while mustard condiment crops include Brassica juncea (L.) Czern. et Coss. and Sinapis alba L. Many species are also important vegetable crops, e.g. Brassica oleracea L. Several species, e.g., Camelina sativa (L.) Crantz, Crambe abyssinica Hochst. ex Fries, and Eruca sativa Mill., have potential as new edible/ industrial oil crops. Many crucifers are grown as ornamentals, e.g., Orychophragmus violaceus (L.) O.E. Schulz, Matthiola incana (L.) R. Br. and others. Over 100 genera have been used for medical purposes in virtue of biological constituents such as sinapine, cardiac glycoside, alkaloids, flavonol, and phenols. The Chinese Pharmacopeia (2010) has admitted several botanical origins, e.g. Raphanus sativus L. (Raphanus Semen), Lepidium apetalum Willd. or Descurainia Sophia (L.) Webb ex Prantl. (Descurainiae Semen or Lepidii Semen), Isatis indigotica Fort. (Isatidis Radix) and so on. Several representatives of the family, including Arabidopsis thaliana (L.) Heynh. and Brassica spp., have achieved the well-accepted status of "model organisms" for genomic studies.

Brassicaceae is a natural family and can be easily distinguished morphologically from species of other flowering families based on its highly conserved and fairly uniform flower architecture. However, the taxonomy of the Brassicaceae has long been controversial because of the often poorly delimited generic boundaries and artificially circumscribed tribes. Several authors have tried to provide a natural system to divide the family of Brassicaceae into tribes or genera (Schulz, 1936; Janchen, 1942; Al-Shehbaz, 1984). The characters traditionally used in these studies are few; they include orientation of the radicle in relation to the cotyledons in the embryo, fruit length-to-width ratio, fruit compression and dehiscence, number of rows of seeds in each locule, trichome type, and features of the nectarines. However, most of the characters considered are subject to convergent evolution, at least on the tribal and subtribal level (Hedge, 1976; Al-Shehbaz, 1984). Within the past two decades, several molecular phylogenetic studies on Brassicaceae (Bailey et al., 2006; Koch et al., 2007; Beilstein et al., 2008; German et al., 2009; Khosravi et al., 2009; Warwick and Hall, 2009; Couvreur et al., 2010; Warwick et al., 2010; German et al., 2011; Goodson et al., 2011) have refined the tribal classification, resurrected several tribes previously misrecognized, added the newly established ones, and adjusted limits of many genera. Despite the substantial progress achieved during the past 20 years along the phylogenetic and 
systematic fronts of the family, many unresolved problems, especially the limits of tribes and the discrimination of species, remain unaddressed.

Plant DNA barcoding has recently emerged as a tool for global species identification and has proven extremely useful for numerous applications such as ecological forensics, identification of traded materials, undertaking identifications where there is a shortage of taxonomic expertise available, and assisting species discovery in some plant groups (reviewed in Hollingsworth et al., 2011). In animals, the mitochondrial cytochrome c oxidase I gene (COI) has been favored in species identification; however, this gene has been precluded as a universal plant barcode because of its generally low rate of nucleotide substitution in plant mitochondrial genomes. Therefore, several candidate markers have been proposed for use in plants, including coding plastid markers (matK, rbcL, rpoB and rpoC1) (Kress et al., 2005; Chase et al., 2007; Lahaye et al., 2008), noncoding spacers (psbA-trnH, atpF-atpH, ITS and ITS2) (Kress and Erickson, 2008; Chen et al., 2010; China Plant BOL Group et al., 2011), or various combinations of several markers (Chase et al., 2007).

Despite efforts to delimitate species and reconstruct the phylogeny of Brassicaceae, little research has been carried out to investigate the applicability and effectiveness of different DNA regions as barcodes to identify species within Brassicaceae. This is especially true for economically important species including edible and industrial oilseed, vegetable, herb, ornamental and fodder crop species. In this study, we utilized five intensively recommended regions ( $r b c \mathrm{~L}, m a t \mathrm{~K}, t r n \mathrm{H}-p s b \mathrm{~A}$, ITS, ITS2) to evaluate their feasibility as candidate DNA barcodes to discriminate economically important Brassicaceae species in China and to establish a new digital identification method.

\section{MATERIAL AND METHODS}

\section{Plant materials}

Plant samples were collected from different locations in China and identified by one of our authors, Prof. Yueyu Hang. In total, 58 individual samples belonging to 27 species, representing a majority of economic species, were collected for further analysis. Fresh leaves were dried in silica gel at the time of collection. Voucher specimens were deposited in the herbarium at the Kunming Institute of Botany, Chinese Academy of Sciences (KIM) (Table 1).

\section{DNA extraction, amplification, and sequencing}

Genomic DNA was extracted following a cetyl trimethylammonium bromide (CTAB) protocol modified from Paterson et al. (2011). The universal primers rbcLa-f and rbcLa-rev (the Consortium for the Barcode of Life (CBOL) recommended), 3F_KIM and 1R_KIM (CBOL recommended), trnH and psbA (Sang et al., 1997), and ITS1 and ITS4 (White et al., 1990) were used in the amplification of $r b c \mathrm{~L}$, matK, $p s b \mathrm{~A}-t r n \mathrm{H}$, and ITS regions respectively.

Polymerase chain reaction (PCR) amplification of the four candidate barcodes was carried out using the following program: a premelt of $3 \mathrm{~min}$ at $94^{\circ} \mathrm{C}$, followed by 35 cycles of $45 \mathrm{~s}$ denaturation at $94^{\circ} \mathrm{C}, 30 \mathrm{~s}$ annealing reaction at $53^{\circ}-58^{\circ} \mathrm{C}$, and finally a $30 \mathrm{~s}$ extension at $72^{\circ} \mathrm{C}$. Each $20-\mu \mathrm{L}$ reaction mixture contained $30 \mathrm{ng}$ genomic DNA template, $2.5 \mathrm{mM} \mathrm{MgCl}_{2}$, $1 \mathrm{X}$ Mg-free DNA polymerase buffer, $0.12 \mathrm{mM}$ dNTPs, $0.3 \mu \mathrm{M}$ of each primer, and $1 \mathrm{U}$ Taq DNA polymerase. PCR products were examined electrophoretically on $0.8-1.2 \%$ agarose gels. Purification and bidirectional sequencing were completed by Beijing Genomics Institute (BGI) using the amplification primers. 







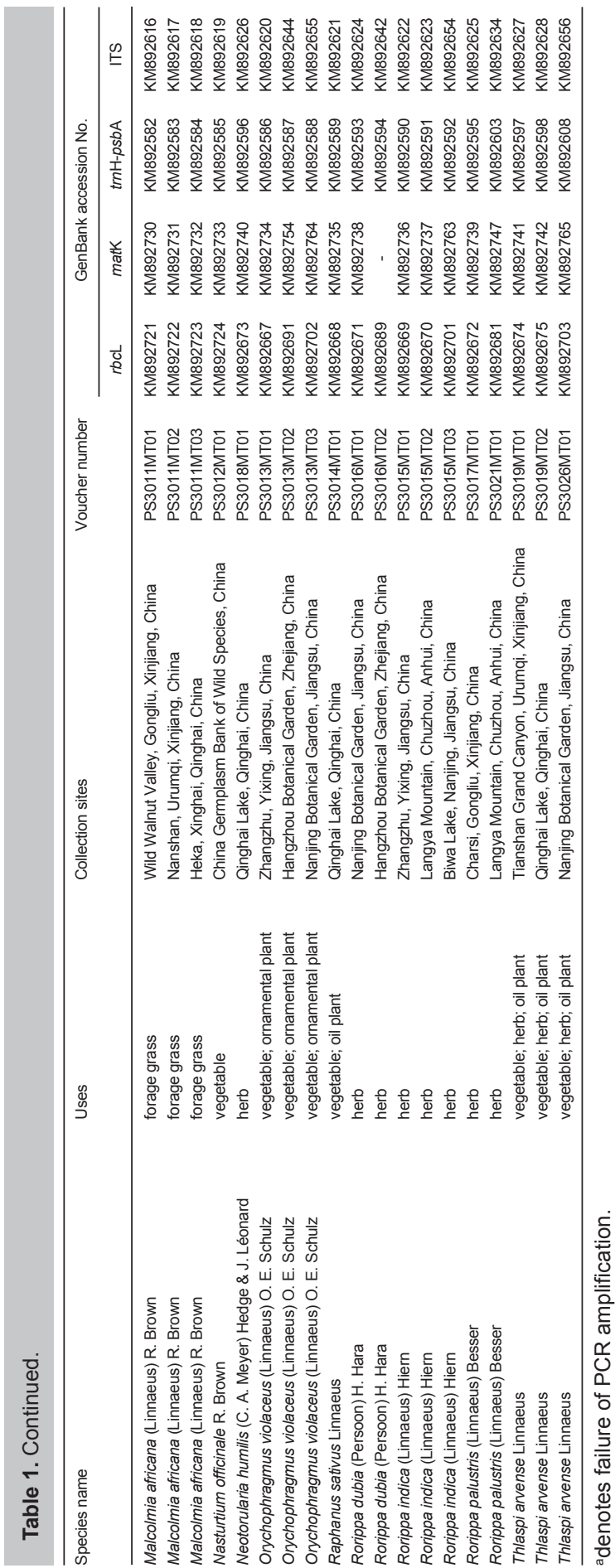




\section{Data analysis}

As the recent intensively recommended DNA barcode candidate, the internal transcribed spacer 2 (ITS2) was also adopted as the fifth region for species discrimination, and the sequences of ITS2 were retrieved according to Keller et al. (2009) and GenBank annotations.

Sequences were aligned and adjusted manually using Sequencer v.4.5 software (GeneCodes, Ann Arbor, MI, USA). The nucleotide sequence data of the four regions were deposited in the GenBank database (Table 1). All genetic distances were calculated using MEGA (5.0 Version) software. Average intra-specific distance, mean theta and coalescent depth were calculated to determine intra-specific variation and average interspecific distance. Theta prime and the minimum interspecific distance were calculated to determine interspecific divergence (Meier et al., 2008; Chen et al., 2010). The distribution of intra-specific versus interspecific variability was evaluated by assessment of the presence of DNA barcoding gaps. Moreover, BLAST 1 and the nearest distance method were used to test the power of species identification as described previously (Sun et al., 2012).

\section{RESULTS}

\section{PCR amplification efficiency and the success rate of sequencing}

The sequence information of the five DNA barcode candidates, $r b c \mathrm{~L}$, matK, $t r n \mathrm{H}-p s b \mathrm{~A}$, ITS and ITS2, is provided in Table 2. The lengths of alignable sequences ranged from $209 \mathrm{bp}$ for ITS2 to $747 \mathrm{bp}$ for matK. rbcL was the most conserved region (529/577 nucleotides), based on both sequence length and number of conserved sites. $t r n \mathrm{H}-p s b \mathrm{~A}$ had the greatest nucleotide variation (233/351), followed by ITS2 (104/209) and ITS (195/517), based on sequence length and number of variable sites. $t r n \mathrm{H}-p s b \mathrm{~A}$ had the richest parsimony (parsim)-informative sites (182/351), followed by ITS2 (84/209), ITS (145/517) and matK (120/747), with rbcL being the lowest. It could be inferred that $t r n \mathrm{H}-p s b \mathrm{~A}$, ITS and ITS2 are the best regions for use as DNA barcodes for phylogenetic reconstruction, whereas $r b c L$ is the least suitable marker for Brassicaceae.

Table 2. Sequence information of five candidate barcodes.
\begin{tabular}{lccccc}
\hline Marker & Sequence length (bp) & Alignment length (bp) & Conserved sites (bp) & Variable sites (bp) & Parsim-informative sites (bp) \\
\hline$r b c$ L & $587-612$ & 577 & 529 & 48 & 33 \\
matk & $773-882$ & 747 & 566 & 181 & 120 \\
trnH- psbA & $253-447$ & 351 & 109 & 233 & 182 \\
ITS & $474-625$ & 517 & 287 & 195 & 145 \\
ITS2 & $181-200$ & 209 & 75 & 104 & 84 \\
\hline
\end{tabular}

$r b c \mathrm{~L}$, matK, trnH-psbA and ITS were all successfully amplified using one pair of universal primers per locus and were compared in the success rates of PCR amplification. As shown in Table $3, r b c \mathrm{~L}$ and ITS displayed the highest efficiency of PCR amplification, followed by $t r n \mathrm{H}-p s b \mathrm{~A}$, with matk being the lowest. 


\begin{tabular}{lcccrr}
\multicolumn{6}{l}{ Table 3. Analysis of inter-specific divergence between species and intra-specific variation. } \\
\hline & matK & $r b c$ & trnH-psbA & ITS & ITS2 \\
\hline All inter-specific distance & $0.0183 \pm 0.0170$ & $0.0028 \pm 0.0043$ & $0.0388 \pm 0.0524$ & $0.0846 \pm 0.0700$ & $0.0878 \pm 0.0702$ \\
Theta prime & $0.0262 \pm 0.0156$ & $0.0034 \pm 0.0042$ & $0.0441 \pm 0.0456$ & $0.0730 \pm 0.0429$ & $0.0860 \pm 0.0437$ \\
Minimum inter-specific distance & $0.0181 \pm 0.0203$ & $0.0025 \pm 0.0033$ & $0.0245 \pm 0.0385$ & $0.0355 \pm 0.0430$ & $0.0419 \pm 0.0541$ \\
All intra-specific distance & $0.0135 \pm 0.0120$ & $0.0012 \pm 0.0035$ & $0.0325 \pm 0.0567$ & $0.0607 \pm 0.0797$ & $0.0706 \pm 0.0972$ \\
Theta & $0.0180 \pm 0.0171$ & $0.0027 \pm 0.0050$ & $0.0403 \pm 0.0583$ & $0.0718 \pm 0.0557$ & $0.0876 \pm 0.0798$ \\
Coalescent depth & $0.0250 \pm 0.0220$ & $0.0035 \pm 0.0058$ & $0.0595 \pm 0.0776$ & $0.1125 \pm 0.0865$ & $0.1346 \pm 0.1124$ \\
Success rate of PCR amplification /\% & 89.66 & 100 & 98.28 & 100 & $-{ }^{\mathrm{a}}$ \\
\hline
\end{tabular}

aITS2 sequences were retrieved from ITS sequences using methods developed by Keller et al., 2009.

\section{Intra-specific variation and inter-specific divergence}

A favorable barcode should possess a high inter-specific divergence to distinguish different species (Gao et al., 2010). Six metrics were used to characterize inter- versus intraspecific variations (Lahaye et al., 2008). ITS2 and ITS exhibited significantly higher inter-specific discrimination than $r b c \mathrm{~L}$, matK and $t r n \mathrm{H}-p s b \mathrm{~A}$. The intra-specific variations were similar, with ITS2 and ITS contributing the largest, and $r b c L$ the smallest variations (Table 3). ITS2 and ITS were found to have high inter-specific divergence and high intra-specific variation, which indicated that ITS2 and ITS could be proposed as the most suitable DNA barcodes to distinguish the species of economic importance in Brassicaceae.

\section{Barcoding gap assessment}

A robust DNA barcode should have separate and non-overlapping genetic variations between intra- and inter-specific samples. The distributions of intra-specific versus inter-specific divergence were examined in the seven barcodes at a scale of 0.001 distance units. Although no distinct barcoding gaps, as typical of $\mathrm{CO} 1$, were found in the distributions of all the loci, the distributions of intra-specific versus inter-specific divergence does suggest a clearly defined range, where the intra-specific variation is considerably lower than the inter-specific divergence (Figure 1). Among them, ITS revealed a relatively well separated distribution, indicating significantly higher inter-specific divergences than their corresponding intra-specific variations, whereas the other four candidate barcodes displayed a distinct overlap without gaps between intra-specific variation and inter-specific divergence.

\section{Identification efficiency of the DNA barcodes}

BLAST 1 and the nearest genetic distance were utilized to assess correct discrimination using different barcodes. The results based on BLAST 1 method indicated that ITS and $\operatorname{trn} \mathrm{H}-$ psbA had the list highest identification efficiency (67.2 and 63.2\%) at the species level, followed by ITS2, matK and $r b c L$. At the genus level, both matK and $r b c L$ had the highest success rate (78.9 and 78.6\%), meanwhile trnH-psbA, ITS and ITS2 also performed well with 76.4, 73.2 and $64.3 \%$ successful identification rates, respectively. Similar results could be obtained by the nearest genetic distance method, while identification efficiency by the nearest genetic distance method was much lower than BLAST 1 (Table 4). 


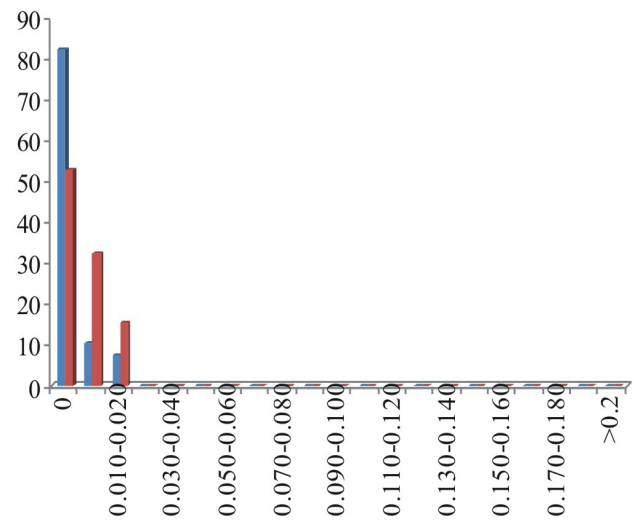

$\operatorname{rbcL}$

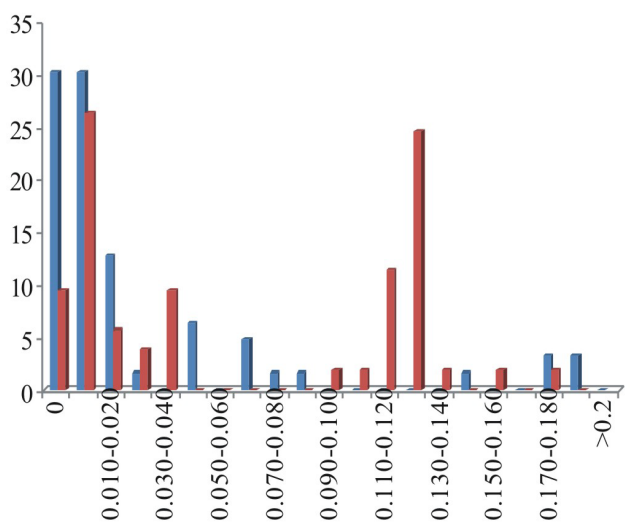

$\operatorname{trn} \mathrm{H}^{-} p \operatorname{sb} \mathrm{A}$

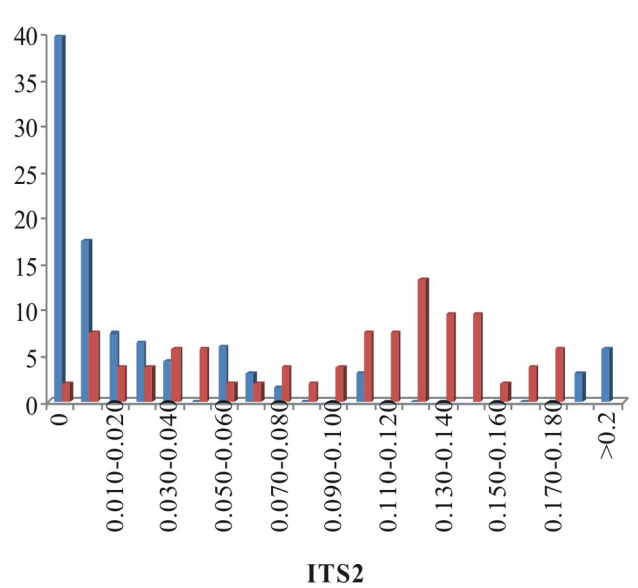

Figure 1. Relative distribution of inter-specific divergence between congenic species and intra-specific variation.
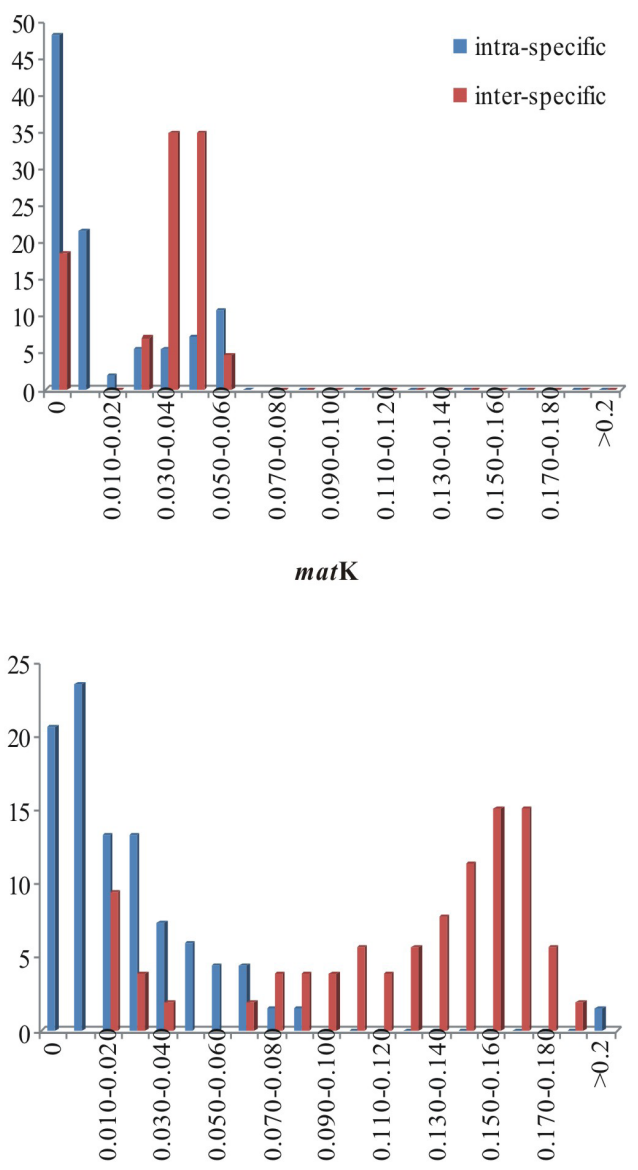

ITS

Genetics and Molecular Research 14 (4): 15050-15061 (2015) 
Table 4. Comparison of identification efficiency for candidate barcodes using different methods of species identification.

\begin{tabular}{|c|c|c|c|c|c|c|c|c|c|}
\hline & \multirow[t]{2}{*}{ Method } & \multirow[t]{2}{*}{ No. of species } & \multirow[t]{2}{*}{ No. of samples } & \multicolumn{2}{|c|}{ Successful identification } & \multicolumn{2}{|c|}{ Incorrect identification } & \multicolumn{2}{|c|}{ Ambiguous identification } \\
\hline & & & & Species & Genus & Species & Genus & Species & Genus \\
\hline \multirow[t]{2}{*}{$r b c \mathrm{~L}$} & BLAST 1 & 27 & 58 & 56.9 & 78.6 & 0 & 0 & 43.1 & 21.4 \\
\hline & Distance & 27 & 58 & 56.9 & 67.9 & 0 & 0 & 43.1 & 32.1 \\
\hline \multirow[t]{2}{*}{ matK } & BLAST 1 & 25 & 52 & 61.1 & 78.9 & 0 & 0 & 38.9 & 21.1 \\
\hline & Distance & 25 & 52 & 61.1 & 71.2 & 0 & 0 & 38.9 & 28.8 \\
\hline \multirow[t]{2}{*}{$t r n \mathrm{H}-p s b \mathrm{~A}$} & BLAST 1 & 27 & 57 & 63.2 & 76.4 & 0 & 0 & 36.8 & 23.6 \\
\hline & Distance & 27 & 57 & 56.1 & 65.5 & 0 & 0 & 43.9 & 34.5 \\
\hline \multirow[t]{2}{*}{ ITS } & BLAST 1 & 27 & 58 & 67.2 & 73.2 & 0 & 0 & 32.8 & 26.8 \\
\hline & Distance & 27 & 58 & 60.3 & 67.9 & 0 & 0 & 39.7 & 32.1 \\
\hline \multirow[t]{2}{*}{ ITS2 } & BLAST 1 & 27 & 58 & 60.3 & 64.3 & 0 & 0 & 39.7 & 35.7 \\
\hline & Distance & 27 & 58 & 53.4 & 62.5 & 0 & 0 & 46.6 & 37.5 \\
\hline
\end{tabular}

\section{DISCUSSION}

\section{Assessment of the applicability of the candidate barcodes}

Several DNA regions, the majority taken from the plastid genome, have been tested for universality and discriminatory power in plants (Kress et al., 2005; Hollingsworth et al., 2009). The two-marker combination of $r b c L+$ matK was proposed as the core barcode for land plants in 2009 (CBOL Plant Working Group, 2009). The Third International Barcoding of Life Conference in Mexico City suggested that a third chloroplast DNA region (cpDNA) (trnH-psbA), and the nuclear ribosomal internal transcribed spacer (ITS) regions, should be treated as complementary loci. Despite the fact that cpDNA regions have been proposed repeatedly for plant barcoding, our results suggest these cpDNA regions cannot establish genetic delimitations between closely related species. This may be attributable to the maternal inheritance of cpDNA in most angiosperms. Thus, plastid variants are only dispersed by seed and cannot travel as far as nuclear alleles, which are dispersed by both pollen and seed (Petit et al., 2005). The limited dispersal of the plastid plant barcodes consequently have a built-in limitation to tracking species boundaries in some cases, which may provide a satisfactory explanation for the low discrimination power of plastid plant barcodes.

As summarized by Hollingsworth (2011), hybridization or polyploid speciation can lead to incongruence between barcode sequences and taxon concepts. Past hybridization or allopolyploidization can lead to shared haplotypes among species (Fazekas et al., 2009). As many as three polyploidization events have occurred in Brassicaceae, with the last one pinpointed asspecific for "core Brassicaceae" (Schranz and Mitchell-Olds, 2006). Consequently, new polyploid species could be generated by autopolyploidy or through inter-species hybridization; for example, Brassica napus $(\mathrm{N}=19)$ was formed by hybridization of $B$. rapa $\mathrm{L}$. $(\mathrm{N}=10)$ and $B$. oleracea $(\mathrm{N}=$ 9). Taxonomic treatment of polyploid derivatives and their respective progenitors is problematic. Moreover, in some cases, the taxonomic groups were poorly defined according to limited morphological characters under convergent evolution, which makes Brassicaceae barcoding an even greater necessity. An ideal DNA barcode should be universal, reliable, cost-effective and show good discriminatory power (CBOL Plant Working Group, 2009). Despite the highest list species-level identification efficiency $(67.2 \%)$ of ITS, in this study, none of the five DNA barcode candidates met all these criteria. 


\section{ITS and ITS2, which is better as an ideal DNA barcode?}

The ITS or its part sequence, ITS2, have been suggested repeatedly as barcodes for plants (Kress et al., 2005; Chen et al., 2010). The ITS generally has a greater discriminatory power over plastid regions at low taxonomic levels; however, three primary concerns have prevented it from being a core plant barcode. Key concerns regarding ITS are 1) incomplete concerted evolution can lead to divergent paralogous copies within individuals, 2) fungal contamination, and 3) difficulties in amplifying and sequencing this region from diverse sample sets. In our analysis, direct sequencing of single-copy ITS sequences was successful in all sampled species (representatives from all three lineages in Brassicaceae), and no fungal contamination was detected. It seems the extent of problems concerning ITS as a core plant DNA barcode is not as pervasive, at least in Brassicaceae, as previously estimated. ITS2 was proposed as an alternative plant barcode with the advantage of easily amplifying and sequencing (Chen et al., 2010; Yao et al., 2010), and it has proven useful in several studies (Gao et al., 2010). Our study revealed the discriminatory power of ITS2 is generally $7 \%$ lower than ITS. Previous studies have suggested that ITS1 and ITS exhibit higher inter-specific divergence relative to ITS2 (Kress et al., 2005). Thus, the use of ITS2 involves a trade-off between using a shorter region of ITS to make recovery and sequencing easier, while sacrificing the number of available characters. Moreover, the preclusion of ITS from being a core plant barcode needs rigorous consideration instead of few formal empirical estimates.

As suggested by Chase and Fay (2009), nuclear genes can provide more information than organelle DNA, which is inherited from only one parent. Multiple attempts have been made to shed light on the way to the ideal plant barcode. Five nuclear low-copy loci (CHS, DET1, COP1, $P G I C 1$, and RPS2) were investigated to discriminate two species of Pugionium (Brassicaceae), while only one locus (DET1) related to flowering regulations was able to delimitate species (Wang et al., 2011). It is proposed that genes related to species isolation ("speciation genes") or linked genes such as mitochondrial DNA (mtDNA) for animals may be more effective in discriminating between closely related species. However, until now, it has been difficult to find such a locus that is universally linked to the speciation of the different plant groups. Recently, 59 low-copy nuclear genes were carefully selected in analysis of angiosperm phylogeny and resulted in highly supported relationships (Zeng et al., 2014), and also highlighted the feasibility of low-copy nuclear loci in the barcoding of plants.

\section{Conflicts of interest}

The authors declare no conflict of interest.

\section{ACKNOWLEDGMENTS}

Research supported by the Ministry of Science and Technology, China, Basic Research Project (\#2013FY112600), the National Natural Science Foundation of China (\#31200177; \#31470448) and Funds of Jiangsu Province Key Laboratory for Plant Ex Situ Conservation (\#QIAN201202) granted to X.Q. Sun. 


\section{REFERENCES}

Al-Shehbaz IA (1984). The tribes of Cruciferae (Brassicaceae) in the southeastern United States. J. Arnold Arbor. 65: 343-373. Bailey CD, Koch MA, Mayer M, Mummenhoff K, et al. (2006). Toward a global phylogeny of the Brassicaceae. Mol. Biol. Evol. 23: $2142-2160$.

Beilstein MA, Al-Shehbaz IA and Kellogg EA (2006). Brassicaceae phylogeny and trichome evolution. Am. J. Bot. 93: 607-619.

Beilstein MA, Al-Shehbaz IA, Mathews S and Kellogg EA (2008). Brassicaceae phylogeny inferred from phytochrome A and ndhF sequence data: tribes and trichomes revisited. Am. J. Bot. 95: 1307-1327.

CBOL Plant Working Group (2009). A DNA barcode for land plants. Proc. Natl. Acad. Sci. U.S.A. 106: 12794-12797.

Chase MW, Cowan RS, Hollingsworth PM, van den Berg C, et al. (2007). A proposal for a standardised protocol to barcode all land plants. Taxon 56: 295-299.

Chase MW and Fay MF (2009). Barcoding of plants and fungi. Science 325: 682-683.

Chen S, Yao H, Han J, Liu C, et al. (2010). Validation of the ITS2 region as a novel DNA barcode for identifying medicinal plant species. PLOS ONE 5: e8613.

China Plant BOL Group, Li DZ, Gao LM, Li HT, et al. (2011). Comparative analysis of a large dataset indicates that internal transcribed spacer (ITS) should be incorporated into the core barcode for seed plants. Proc. Natl. Acad. Sci. U.S.A. 108: $19641-19646$.

Couvreur TL, Franzke A, Al-Shehbaz IA, Bakker F, et al. (2010). Molecular phylogenetics, temporal diversification, and principles of evolution in the mustard family (Brassicaceae). Mol. Biol. Evol. 1: 55-71.

Fazekas AJ, Kesanakurti PR, Burgess KS, Percy DM, et al. (2009). Are plant species inherently harder to discriminate than animal species using DNA barcoding markers? Mol. Ecol. Resour. 9: 130-139.

Gao T, Yao H, Song J, Zhu Y, et al. (2010). Evaluating the feasibility of using candidate DNA barcodes in discriminating species of the large Asteraceae family. BMC Evol. Biol. 10: 324.

German DA, Friesen N, Neuffer B, Al-Shehbaz IA, et al. (2009). Contribution to ITS phylogeny of the Brassicaceae, with special reference to some Asian taxa. Plant Syst. Evol. 283: 33-56.

German DA, Grant JR, Lysák MA and Al-Shehbaz IA (2011). Molecular phylogeny and systematics of the tribe Chorispora (Brassicaceae). Plant Syst. Evol. 294: 65-86.

Hedge IC (1976). A systematic and geographical survey of the Old World Cruciferae. In: The biology and chemistry of the Cruciferae (Vaughan JG, MacLeod AJ and Jones BMG, eds.). Academic Press, London, 1-145.

Hollingsworth ML, Andra Clark A, Forrest LL, Richardson J, et al. (2009). Selecting barcoding loci for plants: evaluation of seven candidate loci with species-level sampling in three divergent groups of land plants. Mol. Ecol. Resour. 9: 439-457.

Hollingsworth PM, Graham SW and Little DP (2011). Choosing and using a plant DNA barcode. PLOS ONE 6: e19254.

Janchen E (1942). Das System der Cruciferen. Oesterreichische Botanische Zeitschrift 91:1-28.

Keller A, Schleicher T, Schultz J, Müller T, et al. (2009). 5.8S-28S rRNA interaction and HMM-based ITS2 annotation. Gene 430: $50-57$.

Khosravi AR, Mohsenzadeh S and Mummenhoff K (2009). Phylogenetic relationships of Old World Brassicaceae from Iran based on nuclear ribosomal DNA sequences. Biochem. Syst. Ecol. 37: 106-115.

Koch MA, Dobes C, Kiefer C, Schmickl R, et al. (2007). Supernetwork identifies multiple events of plastid trnF(GAA) pseudogene evolution in the Brassicaceae. Mol. Biol. Evol. 24: 63-73.

Kress WJ and Erickson DL (2008). DNA barcodes: genes, genomics, and bioinformatics. Proc. Natl. Acad. Sci. U.S.A. 105: 2761-2762.

Kress WJ, Wurdack KJ, Zimmer EA, Weigt LA, et al. (2005). Use of DNA barcodes to identify flowering plants. Proc. Natl. Acad. Sci. U.S.A. 102: 8369-8374.

Lahaye R, van der Bank M, Bogarin D, Warner J, et al. (2008). DNA barcoding the floras of biodiversity hotspots. Proc. Natl. Acad. Sci. U.S.A. 105: 2923-2928.

Meier R, Zhang GY and Ali F (2008). The use of mean instead of smallest interspecific distances exaggerates the size of the "barcoding gap" and leads to misidentification. Syst. Biol. 57: 809-813.

Paterson AH, Brubaker CL and Wendel JF (1993). A rapid method for extraction of cotton (Gossypium spp) genomic DNA suitable for RFLP of PCR analysis. Plant Mol. Biol. Rep. 11: 122-127.

Petit RJ, Duminil J, Fineschi S, Hampe A, et al. (2005). Comparative organization of chloroplast, mitochondrial and nuclear diversity in plant populations. Mol. Ecol.14: 689-701.

Pharmacopoeia Commission of People's Republic of China. (2010) Pharmacopoeia of People's Republic of China. Part 1. Chemical Industry Press, Beijing, 191: 255-314.

Ran JH, Wang PP, Zhao HJ and Wang XQ (2010). A test of seven candidate barcode regions from the plastome in Picea (Pinaceae). J. Integr. Plant Biol. 52: 1109-1126. 
Sang T, Crawford DJ and Stuessy TF (1997). Chloroplast DNA phylogeny, reticulate evolution, and biogeography of Paeonia (Paeoniaceae). Am. J. Bot. 84: 1120.

Schranz ME and Mitchell-Olds T (2006). Independent ancient polyploidy events in the sister families Brassicaceae and Cleomaceae. Plant Cell 18: 1152-1165.

Schulz OE (1936). Cruciferae. In: Die Natürlichen Pflanzenfamilien, vol 17B, 2nd edn. (Engler A, Harms H, eds.) Verlag von Wilhelm Engelmann, Leipzig, 227-658.

Sun XQ, Zhu YJ, Guo JL, Peng B, et al. (2012). DNA barcoding the Dioscorea in China, a vital group in the evolution of monocotyledon: use of matK gene for species discrimination. PLoS One 7: e32057.

Wang Q, Yu QS and Liu JQ (2011). Are nuclear loci ideal for barcoding plants? A case study of genetic delimitation of two sister species using multiple loci and multiple intraspecific individuals. J. Syst. Evol. 49: 182-188.

Warwick SI and Hall JC (2009). Phylogeny of Brassica and wild relatives. In: Biology and breeding of crucifers (Gupta S, eds.). CRC Press, New York, 19-36.

Warwick SI, Mummenhoff K, Sauder CA, Koch MA, et al. (2010). Closing the gaps: phylogenetic relationships in the Brassicaceae based on DNA sequence data of nuclear ribosomal ITS region. Plant Syst. Evol. 285: 209-232.

White TJ, Bruns TD, Lee SB and Taylor JW (1990). Amplification and direct sequencing of fungal ribosomal RNA genes for phylogenetics. In: PCR protocols: a guide to methods and applications (Innis MA, Gelfand DH, Sninsky JJ and White TJ, eds.). Academic Press, London, 315-322.

Yao H, Song J, Liu C, Luo K, et al. (2010). Use of ITS2 region as the universal DNA barcode for plants and animals. PLoS One 5: e13102.

Zeng LP, Zhang Q, Sun RR, Kong HZ, et al. (2014). Resolution of deep angiosperm phylogeny using conserved nuclear genes and estimates of early divergence times. Nat. Commun. 5: 4956. 